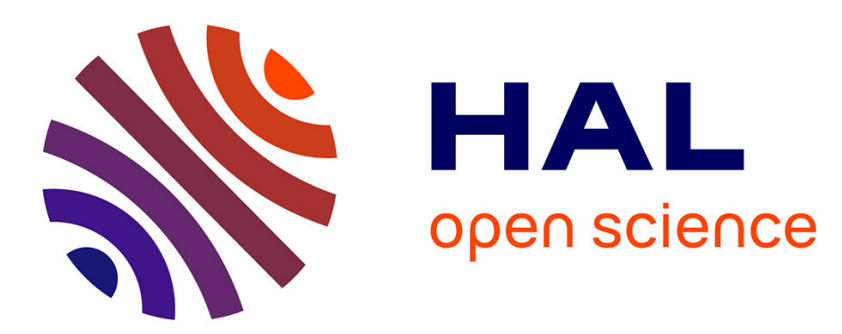

\title{
Experimental control of steady state photorefractive self-focusing in InP:Fe at infrared wavelengths
}

\author{
Cristian Dan, Delphine Wolfersberger, Nicolas Fressengeas
}

\section{To cite this version:}

Cristian Dan, Delphine Wolfersberger, Nicolas Fressengeas. Experimental control of steady state photorefractive self-focusing in InP:Fe at infrared wavelengths. Applied Physics B - Laser and Optics, 2011, 104 (4), pp.887-895. 10.1007/s00340-011-4438-y · hal-00559841

\section{HAL Id: hal-00559841 \\ https://hal.science/hal-00559841}

Submitted on 2 Feb 2011

HAL is a multi-disciplinary open access archive for the deposit and dissemination of scientific research documents, whether they are published or not. The documents may come from teaching and research institutions in France or abroad, or from public or private research centers.
L'archive ouverte pluridisciplinaire HAL, est destinée au dépôt et à la diffusion de documents scientifiques de niveau recherche, publiés ou non, émanant des établissements d'enseignement et de recherche français ou étrangers, des laboratoires publics ou privés. 


\title{
Experimental control of steady state photorefractive self-focusing in InP:Fe at infrared wavelengths
}

\author{
C. Dan, D. Wolfersberger and N. Fressengeas \\ Laboratoire Matériaux Optiques, Photonique et Systèmes \\ Unité de Recherche Commune à l'Université de Metz et Supélec \\ 2, rue Edouard Belin, 57070 Metz Cedex, France
}

January 31, 2011

\begin{abstract}
This paper reports an experimental study of the self-focusing process in iron doped indium phosphide at an 1.06 micron wavelength, identifying the influence of temperature, beam intensity and background illumination for two different iron dopings. We point out that the iron ionization ratio is at the origin of different qualitative behavior previously reported and we show that it is possible to reproduce the said behaviors in the same crystal by applying a uniform illumination, allowing their eventual control for dynamic wave-guiding.
\end{abstract}

\section{Introduction}

Self focusing of a laser beam in a photorefractive (PR) materials is a process that leads to the formation of waveguides in bulk crystals. Under the right conditions, such a self focused beam can propagate as a spatial soliton $[1,2]$. Since their first observation $[3,4]$, the PR solitons have been extensively characterized from experimental and theoretical point of view. As such, their properties are well known in typical PR materials (such as $\mathrm{SBN}, \mathrm{Bi}_{12} \mathrm{TiO}_{20}$ and $\mathrm{BaTiO}_{3}\left[5_{-}\right.$ $8]$ ), in which the PR effect is due mainly to only one type of charge carriers and occurs at visible wavelengths. The dynamics of self focusing and soliton formation have been studied and characterized at steady state [9-12] as well as in transient regime $[13,14]$, together with its dependency on various parameters (temperature, background illumination) for two different iron dopings.

Spatial solitons are especially interesting for all optical routing/switching applications, since they allow the inscription of waveguides in 3D inside bulk crystals for relatively low intensities[15]. Moreover, two self focused beams can interact [16-20], allowing to control the propagation direction of one beam with another beam, thus giving the basic mechanism for an all optical router. 
Guides created by PR self focusing are easily erasable, which allows the creation of dynamic waveguides. On the other hand, it is also possible to create long lasting waveguides by 'fixing' them in place [21,22]. More recently, the control of arrays of PR solitons has been demonstrated [23].

For the above mentioned reasons, PR self focused beams are a promising candidate for applications involving the dynamic guiding of light in 3D. However, one of their major drawbacks is the slow response time, ranging from seconds [14] to tens of minutes [24]. Response times on the order of nanoseconds can be reached $[25,26]$, but for intensities several orders of magnitudes higher than those usually available in telecommunications. In order to circumvent this disadvantage, we have turned our attention to PR semiconductors, namely the iron doped indium phosphide (InP:Fe). Indeed, it has been already proven that, in semiconductors, waveguides can be inscribed much faster for relatively low intensities: measured response times are of the order of milliseconds in tin hypotiodiphosphate $\left(\mathrm{Sn}_{2} \mathrm{P}_{2} \mathrm{~S}_{6}\right)$ [27] or even microseconds in InP:Fe [28], opening the way for telecommunication wavelength routing applications [29]. Moreover, semiconductors such as InP, CdTe or $\mathrm{Sn}_{2} \mathrm{P}_{2} \mathrm{~S}_{6}$ can be rendered sensitive to infrared (IR) wavelengths by appropriate doping.

However, the first experimental observation of PR self focusing in InP:Fe [30] showed that its behavior is significantly different from that described by the 'classical' models mentioned above. For instance, in InP:Fe[31-34], or $\mathrm{CdZnTe}[35]$ the space charge field created by the PR effect can be several times higher than the external applied field. This 'amplification' of the electric field compensates for the lower electro-optic coefficient of the semiconductors, allowing the creation of an efficient waveguide [33]. Another particularity is that in semiconductors the focusing process switches from self defocusing to self focusing when the intensity of the beam increases, while in 'classical' materials the beam always focuses (or defocuses, depending on electric field direction), regardless of its intensity. At the origin of these differences is the fact that, in semiconductors, the PR effect is due to two types of charge carriers, whereas in the previously used materials the PR effect is produced by only one type of charge carriers.

Naturally, theoretical models have been developed in order to characterize the PR self focusing in semiconductors, all of them within the linear transport approximation. Until recently, none of them were able to fully explain the range of experimental results obtained. The first models [30] stem from two wave mixing (TWM) theory and are based on some limiting assumption specific to TWM (such as a periodic weakly modulated illumination grating) which are not valid in the self focusing experiments where only one intense beam is used. Moreover, they are considering the steady state of the self focusing only. A later model $[28,36]$ does take into account the transient regime of the self focusing, but it does not explain the electric field amplification observed experimentally. Also, in [36] no transition from self defocusing to self focusing is observed when the intensity of the beam is increased.

The limitations of previous theories have been circumvented by two new generalized models $[37,38]$. One of them $([37])$ is a time-resolved numerical analysis 
of the propagation of a $2 \mathrm{D}$ beam in an InP:Fe crystal, which allows to compute the diameter and the bending of the self focused beam after propagating a given distance. The second one ([38]) analyzes the case of low non-harmonic illumination and it emphasizes the role played by a uniform background intensity in the space charge field creation.

The experimental results presented in this paper allow us to point out the origin of the different behaviors reported in the literature $[30,36]$. In fact, we are able to reproduce the two main types of self focusing (with and without a transition from defocusing to focusing, as presented in [30] and [36] respectively) in the same crystal by using a uniform background illumination. Further on, we show how beam bending and self focusing itself can be controlled by tuning various parameters: iron doping, temperature and background illumination. We also compare our experimental results with the theoretical prediction taken from $[37,38]$.

\section{Experimental setup}

We are using the typical setup employed to characterize PR self focusing: an infrared CW beam with a wavelength of $1.06 \mu \mathrm{m}$ is focused to a $25 \mu \mathrm{m}$ waist on the input face of an InP:Fe crystal. The output face of the crystal is imaged on a CCD camera, thus allowing the measurement of the emergent beam diameter. The beam propagates along $<\overline{1} 10>$ axis and is polarized along $<110>$ axis. A $10 \mathrm{kV} / \mathrm{cm}$ external electric field is applied along the $<001>$ axis. Note that this is the same configuration as the one used in references [30, 36, 37]. The crystal is thermally stabilized with a Peltier cell. The beam peak intensity ranges from $0.01 \mathrm{~W} / \mathrm{cm}^{2}$ to $30 \mathrm{~W} / \mathrm{cm}^{2}$.

Different InP:Fe samples were used: they have the same size $10 \times 5 \times 5 \mathrm{~mm}^{3}$ along the axes $<\overline{1} 10>\times<110>\times<001>$ respectively, but different Fe doping. Their doping was measured by SIMS (Secondary Ion Mass Spectroscopy) method. The measurements gave a Fe concentration of $8 \times 10^{16} \mathrm{~cm}^{-3}$ for the less doped crystal (which we will refer to as 'Type 1') and $10^{17} \mathrm{~cm}^{-3}$ for the more doped crystal ('Type 2'). We have also estimated the doping by the method proposed in [39]. The measured absorption coefficient at $1 \mu \mathrm{m}$ are $0.58 \mathrm{~cm}^{-1}$ (Type 1) and $0.93 \mathrm{~cm}^{-1}$ (Type 2), from which we estimated a Fe concentration of $7.4 \times 10^{16} \mathrm{~cm}^{-3}$ and $9.1 \times 10^{16} \mathrm{~cm}^{-3}$ respectively. These values are in good agreement with SIMS results.

Figure 1 shows typical results obtained at various beam intensities ${ }^{1}$ and for different directions of the applied field. Each image shows the transversal profiles of the beam at the output face of the crystal in the absence and in the presence of an external applied electric field. Without applied field, the beam size is determined by linear diffraction only, while in the presence of the field its size is due to both linear diffraction and nonlinear PR effect. From fig. 1 it is clear that, for a given direction of the electric field (figures (a),(b),(c)), the

\footnotetext{
${ }^{1}$ In this work, by 'beam intensity' we understand the peak intensity in the center of the beam.
} 
beam is defocused at low intensities and focused at high intensities. By changing the direction of the electric field (figures (d),(e),(f)), this behavior is reversed: self-focusing occurs at low intensities, while at higher intensities the beam defocuses. We consider the electric field as positive if it leads to a self-focusing at high intensities and to a defocusing at lower intensities. By this convention, the field is positive in figures (a),(b),(c) and negative in (d),(e),(f).

In order to quantify the self focusing, we define the self focusing ratio (SF) as the ratio between the diameters of the beam at the output face of the crystal with and without an applied external field ${ }^{2}$. As such, a $\mathrm{SF}>1$ indicates a defocusing of the beam, while a $\mathrm{SF}<1$ corresponds to a focusing. The beam diameter is measured by fitting a Gaussian profile to the beam profile and measuring the diameter at an intensity equal to $I_{\max } / e^{2}$, where $I_{\max }$ is the intensity at the center of the beam. This fitting is meaningful most of the time because the Gaussian profile is mostly conserved.

However, as will be shown in the following, beam break up is also observed, for which no beam diameter measurement is meaningful. Furthermore, one should keep in mind that all beam diameter measurements reported in the following are done using this procedure. Though our accuracy estimate for this measure is with a $10 \%$ error margin, the fact that most measured profiles are not exactly Gaussian is probably the main source of measurement errors.

The experimental measurements presented are one shot measurements each time, as an automation of these photorefractive processes is not easy. However, as can be guessed from [28] in which the same measurements are done on a different sample of the same composition crystal, the measurements are well reproducible and we can estimate the measurement error below $10 \%$, everywhere the measurement is meaningful.

\section{$3 \quad$ Experimental results}

\subsection{Focusing behavior vs. beam intensity}

As previously mentioned, SF dependency on the beam intensity is one of the most used methods to characterize PR self focusing, especially when experimental results are compared against simulations. Thus, we have studied the evolution of the SF ratio as a function of beam intensity. As Fig. 2 shows, in both cases we observe a clear transition from defocusing to focusing (for a positive filed) or from focusing to defocusing (for a negative field). We call inversion intensity $\left(I_{\mathrm{inv}}\right)$ the beam intensity at which this transition takes place.

\subsection{Focusing behavior and beam bending vs. temperature}

The beam intensity is the main parameter that determines the self focusing behavior. This is to be expected, since photo excited electrical charges are

\footnotetext{
${ }^{2}$ In the absence of the electric field, the beam diameter at the output face of the crystal is results exclusively of its linear diffraction inside the crystal.
} 

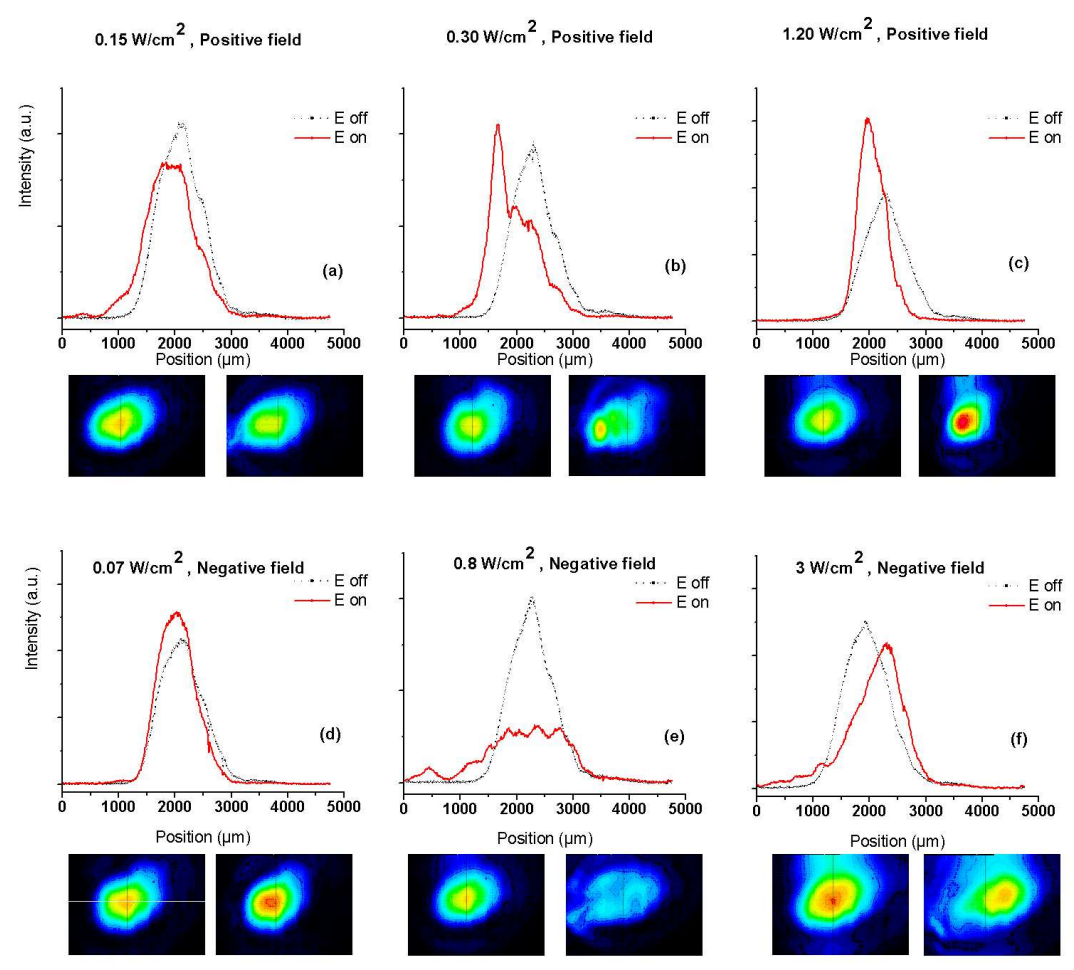

Figure 1: Typical result: comparison between beam profiles at the output of the crystal with and without applied field for different intensities. The output face is imaged on the CCD camera via a 20x magnification. The field is positive in figures (a),(b),(c) and negative in (d),(e),(f). A Type 2 crystal was used (a different sample of the same composition as in [28]). Beam waist at the input face of the crystal is $25 \mu \mathrm{m}$, temperature is $25^{\circ} \mathrm{C}$. Images below each plot show the output beam at the output of the crystal without (left) and with (right) an applied electric field. 


\section{Self focusing ratio vs. beam intensity}

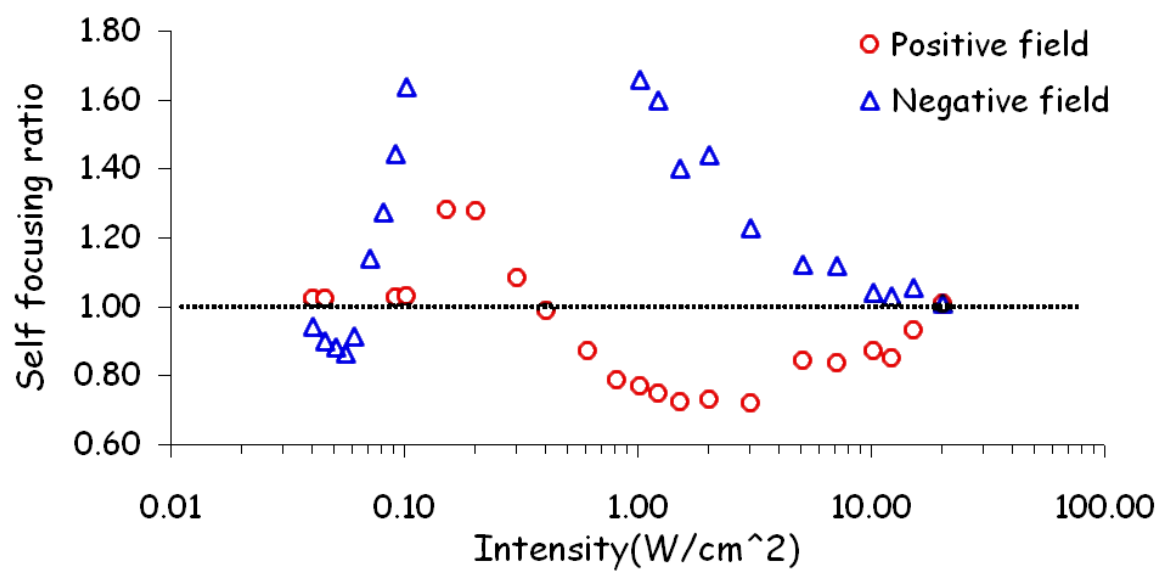

Figure 2: Self focusing ratio vs. beam intensity for positive and negative applied field. Experimental parameters: Type 2 crystal, temperature $20^{\circ} \mathrm{C}$, wavelength $1.06 \mu \mathrm{m}$.

responsible for the PR effect. However, charges are also excited thermally. Therefore, temperature plays also a role in the self focusing process, albeit more subtle. This is equally true for materials with 2 types and 1 type of charge carriers. In previous theoretical models, self focusing is characterized by two specific parameters: the dark intensity $\left(I_{d}\right)$ in [36] and the (resonance intensity $\left(I_{\text {res }}\right)$ in $[30]$.

The former, $I_{d}$, is the intensity which generates as much free carriers as temperature induced thermal excitation. It allows to readily compare thermal and optical excitation by comparing dark and optical intensities. The latter, $I_{\text {res }}$, is the intensity at which photorefractive wave mixing is enhanced in semiconductors in which both elecrons and holes play a significant role. Microscopically speaking, it corresponds to the intensity at which holes and electrons excitation rates are equal, the former being mostly optical and the latter mostly thermal in InP:Fe.

Both these two characteristic intensities depend on the temperature ${ }^{3}[31,36]$ and are directly proportional to it. We must point out that in a most recent theoretical model [37], neither $I_{d}$ nor $I_{\text {res }}$ appear to play an explicit role in the self focusing process. Therefore, we have decided to investigate the influence of the temperature on the self focusing process by studying the evolution of SF ratio vs. intensity at different temperatures. The results are presented in fig. 3 .

SF ratio aside, another parameter essential for controlling the self focusing

\footnotetext{
${ }^{3}$ This dependence is due to the thermal emission rate of the electrons, which both $I_{d}$ and $I_{\text {res }}$ are depending on
} 

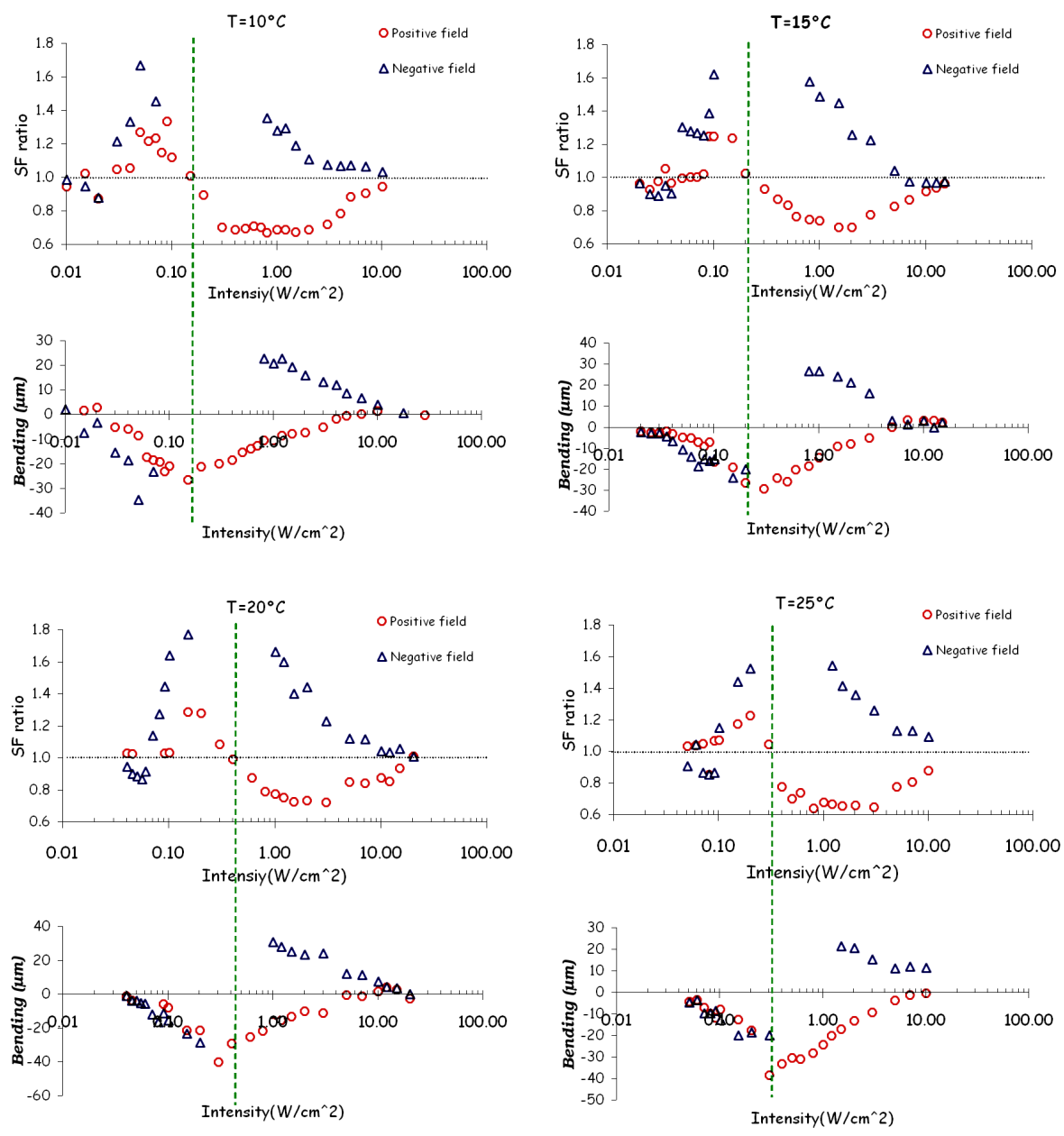

Figure 3: SF ratio and beam bending in a type 2 crystal as a function of beam intensity and direction of applied field at different temperatures: $10^{\circ} \mathrm{C}$ (top-left), $15^{\circ} \mathrm{C}$ (top-right), $20^{\circ} \mathrm{C}$ (bottom-left) and $25^{\circ} \mathrm{C}$ (bottom-right). Horizontal dotted lines correspond to $\mathrm{SF}$ ratio $=1$. Vertical dashed lines indicate $I_{\mathrm{inv}}^{+}$. 
process is the beam bending, especially when dealing with interactions between two or more beams. Thus, we have measured the beam bending as a function of intensity and temperature (Fig. 3). One can see that, for a positive field, the beam deviates always in the same direction ${ }^{4}$ and the maximum bending is reached at $I_{\text {inv }}$. For a negative field and low intensities, the bending has the same direction as for a positive field. Up to this point, these results are in full agreement with [30]. However, one can see that for a negative field and higher intensities the bending becomes positive. This behavior was not observed in [30], most probably due to the break up of the beam that we have observed for negative fields (fig. 1(e)) and that we will discuss in the next section. It is interesting to note that the inversion intensity for a positive field $I_{\text {inv }}^{+}$plays also a role when a negative field is applied. Specifically: for intensities lower than $I_{\text {inv }}^{+}$the bending is always negative, while for intensities higher than $I_{\text {inv }}^{+}$it is always positive. This is the case for the whole temperature range used in our measurements, as seen in Fig. 3.

\subsection{Influence of doping and background illumination}

The influence of a uniform background illumination is well studied and understood in materials with one type of charge carriers. Such a uniform illumination changes the value of $I_{d}$ and, through it, the behavior of the self focusing process. On the other hand, in materials with two type of charge carriers its role has not been systematically studied until recently. A new theoretical study [38] shows that the background intensity is expected to play a crucial role in the self focusing process in InP:Fe. Also, experimental results obtained in another PR semiconductor, the CdZnTe, show that the deflection of a laser beam can be controlled via a background illumination [40]. Thus, we have decided to investigate the influence of a uniform illumination on the self focusing process in our InP:Fe crystals.

The initial $1.06 \mu \mathrm{m}$ beam was split via a 50:50 beam splitter. One of the beam was magnified by a 20x microscope objective and its central part collimated with a lens to a $4 \mathrm{~cm}$ diameter. The expanded beam was then sent on the InP:Fe crystal from above, thus propagating along the $<110>$ axis. We have investigated the evolution of the SF ratio and beam bending for two differently doped crystals with and without background illumination.

In the absence of a uniform illumination, the observed behavior (Fig. 4) is identical to the one depicted in 2: the beam is defocused at low intensities and focused at higher intensities for both crystals, with a maximum bending at $I_{\text {inv }}$. In the presence of a background intensity $\mathrm{I}_{b}=0.015 \mathrm{~W} / \mathrm{cm}^{2}$, the behavior is completely different: the focusing/defocusing transition cannot be observed anymore. Instead, the SF ratio reaches a maximum for a given intensity and

\footnotetext{
${ }^{4} \mathrm{By}$ convention, the bending is positive if the beam moves to the right when an electric field is applied and negative if it moves to the left. In our experimental configuration, a field is positive when applied from left to right, thus for a positive field the beam bending goes in the same direction as the applied field.
} 

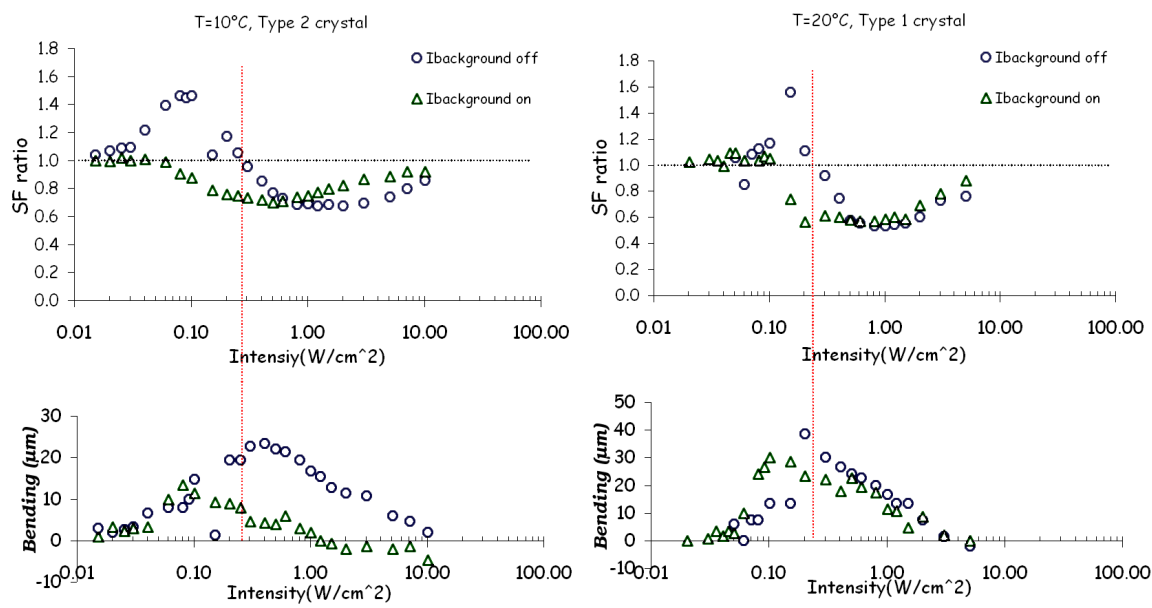

Figure 4: SF ratio vs. beam intensity with and without a uniform background illumination for a type 2 crystal (left) and for a type 1 crystal (right). Temperature is $10^{\circ}$ for type 2 crystal and $20^{\circ}$ for type 1 crystal. A $10 \mathrm{kV} / \mathrm{cm}$ positive electric field was applied. The background illumination intensity is $\mathrm{I}_{b}=0.015$ $\mathrm{W} / \mathrm{cm}^{2}$. Horizontal dotted lines correspond to $\mathrm{SF}$ ratio $=1$. Vertical lines indicate $I_{\text {inv }}^{+}$.

decreases for intensities below and above this value. This behavior is similar to the one described in [36].

\subsection{Beam Breakup}

In all the experimental results presented here, we can note that some experimental points are missing for negative applied fields (see for instance figure 2). This is due to the fact that, for these particular intensities, the beam suffers a complete breakup (as it can be seen in fig. 1(e)). In this case the beam profile is not Gaussian anymore, and therefore is not possible to measure a meaningful diameter of the beam.

We must point out that a similar behavior (with an even more complete breakup of the beam) has been observed experimentally at a wavelength of 1.04 $\mu \mathrm{m}[30]$; in this case $I_{\text {inv }}$ for a negative field ${ }^{5}$ could not been observed directly due to the beam breakup.

\footnotetext{
${ }^{5}$ In reference [30], the sign of the electric field is determined by comparing its orientation with the axes of the crystal. As such, a positive field by this convention corresponds to a negative field in our case.
} 


\section{Discussion and comparison to previous results}

We will start discussing the previously presented results by pointing out that $I_{\text {inv }}$ depends on the direction of the applied field. This section will thus focus on the analysis of the behavior of both values of $I_{\mathrm{inv}}$, corresponding to positive and negative applied field, with respect to the temperature and the background illumination. We will finally conclude by establishing that the influence of this two parameters have strong common points that their share with the doping level.

\subsection{Temperature}

As one can see, the behavior previously observed in Fig.2 repeats consistently for the temperature range $10^{\circ}-25^{\circ} \mathrm{C}$. From these results we can immediately measure the inversion intensity $I_{\text {inv }}$ for a positive field at each temperature:

- $0.14 \mathrm{~W} / \mathrm{cm}^{2}$ at $10^{\circ} \mathrm{C}$.

- $0.2 \mathrm{~W} / \mathrm{cm}^{2}$ at $15^{\circ} \mathrm{C}$.

- $0.4 \mathrm{~W} / \mathrm{cm}^{2}$ at $20^{\circ} \mathrm{C}$.

- $0.3 \mathrm{~W} / \mathrm{cm}^{2}$ at $25^{\circ} \mathrm{C}$.

Based on the 4 above values and the facts that those measurements are highly reproducible, we are able to make a statement about $I_{\text {res }}$ and the role it plays in the self focusing process. Indeed, accordingly to [30], the transition focusing/defocusing occurs precisely at $I_{\text {res }}$. In other words, this means that $I_{\text {res }}=I_{\text {inv }}$. As previously stated, $I_{\text {res }}$ is proportional to the temperature, which is to say that a temperature increase would lead to higher $I_{\text {res }}$. However, from our measurements it is clear that $I_{\text {inv }}\left(25^{\circ} \mathrm{C}\right)>I_{\text {inv }}\left(20^{\circ} \mathrm{C}\right)$. We must therefore conclude that $I_{\text {res }} \neq I_{\mathrm{inv}}$ and that $I_{\text {res }}$ alone is not enough to determine the intensity at which the inversion occurs. This conclusion is in agreement with theoretical results presented in [38], which indicate that $I_{\text {res }}$ and $I_{\text {inv }}$ are indeed different.

By comparing our experimental results with the beam bending predicted by the simulation (see Fig. 5 in ref. [37], one can remark a qualitative disagreement between the two ${ }^{6}$. Namely, for a positive field the simulation predicts a maximum bending of the beam at the intensity for which the SF ratio is minimum, while the experiment shows that the maximum bending always occurs at $I_{\mathrm{inv}}{ }^{7}$. At this moment, it is not clear what is the origin of this disagreement.

\footnotetext{
${ }^{6}$ In this paper we are focusing mainly on qualitative analysis of the differences between theory and experiment. This is due to two factors. On one hand, some of InP:Fe parameters are known with a relatively low precision (this is the case for ionization ratio or photo and thermal excitation cross section). On the other hand, the theoretical model shows that the self focusing behavior is very sensitive to small variations of the input parameters.

${ }^{7}$ We remind that at this intensity, SF ratio is always 1.
} 


\subsection{Background illumination}

Based on the results presented in section 3.3, we conclude that it is possible to reproduce the qualitatively different behaviors reported in the literature by switching on and off a uniform background illumination of the crystal. Note that this is the case for two significantly different iron dopings used in our measurements: as fig. 4 shows, the transition defocusing/focusing disappears for both Type 1 and Type 2 crystals when a background illumination is applied.

In order to find the origin of this behavior, we will have a closer look at the effect that a uniform illumination has on our $\mathrm{crystal}^{8}$. Any illumination will produce a ionization of the $\mathrm{Fe}$ atoms, therefore reducing the concentration of $\mathrm{Fe}^{3+}$ (noted $\mathrm{p}_{t 0}$ ) and increasing the concentration of $\mathrm{Fe}^{2+}$ (noted $\mathrm{n}_{t 0}$ ). By illuminating the entire crystal, the net effect is equivalent increase of the ionization ratio $n_{t 0} / p_{t 0}$. The value of the ionization ratio in InP:Fe crystal in absence of any illumination ranges from $0.04[41]$ to $0.3[42,43]$. However, one has to be aware that the previously mentioned values have not been obtained from direct measurement, but from fitting the ionization ratio to experimental results of TWM experiments. As such, the above estimations are not extremely precise, but nevertheless it seems that InP:Fe crystals can have very different ionization ratio. This is most probably due to impurities and defects introduced during the fabrication process, which lead to different ionization ratio between crystals originating from different manufacturers ${ }^{9}$.

\subsection{Analysis}

As previous simulation showed [37], during the self focusing process in InP:Fe the incident beam induces not only a waveguide, but also an anti-waveguide which goes along the waveguide ${ }^{10}$. Based on this, it becomes clear that the state of the beam at the output of the crystal depends mainly of the way it couples to this guide/anti-guide pair: we observe a focusing if the beam is coupled in the guide or a defocusing if it is 'coupled' by the anti-guide. However, to which one the coupling occurs is not just a matter of their relative position with respect to the beam, but it depends also on their relative sizes and 'depths'11. Actually, at intensities close to $I_{\mathrm{inv}}$ it is possible to couple the beam to both of them, as evidenced in fig. 1(b). By comparing the profiles with and without applied field, one can see that the beam is slightly larger at its base (defocused), but it is clearly focused and deviated at its top ${ }^{12}$.

\footnotetext{
${ }^{8}$ We must point out that the experimental results presented in this paper are not directly comparable to the model presented in [38], since in our case the background intensity is lower than the beam intensity.

${ }^{9}$ Note that crystals used in this paper originate from the same manufacturer, InPact society

${ }^{10} \mathrm{As}$ a side note, we would like to point out that the index contrast between the guide and the anti-guide is responsible for the higher than expected index variation previously measured [33].

11 'Depth' meaning the variation of the refractive index in the guide/anti-guide with respect to the refractive index in the bulk crystal

${ }^{12}$ Around $I_{\text {inv }}$ the beam profile is not Gaussian anymore. Unlike the previously mentioned beam breakup, in this case diameter measurements are still meaningful. However, since the
} 
As previous simulation have shown [37], during the self focusing process in InP:Fe, the incident beam induces not only a waveguide, but also an antiwaveguide which goes along the waveguide ${ }^{13}$. In other words, alongside the waveguide index hump which tends to focus the beam, there lies an almost equivalent index groove which tends to de-focus it. Therefore, the state of the beam at the output of the crystal depends mainly on the way it interacts with this guide/anti-guide pair: we observe a focusing effect if the beam is coupled in the guide or a defocusing one if it mainly interacts with the anti-guide. However, whether it is the guide or the anti-guide that mostly interacts with the beam is not, as could be initially thought, only a matter of initial relative position of guide and beam at the crystal entrance. It also strongly depends on the relative widths of the beam, index hump and groove, as well as the relative index modulation associated to the latter two.

As such, it becomes clear that for a positive applied field and intensities below $I_{\text {inv }}$ the beam is 'coupled' to the anti-guide (leading to defocusing), while for intensities above $I_{\mathrm{inv}}$ it is coupled to the guide (leading to focusing). The same happens for a negative field, only that the focusing occurs at low intensities and the defocusing at higher intensities.

By looking at the parameters we have tuned in our experiments - temperature, doping and ionization ratio (background illumination) - one might be tempted to draw the conclusion that the ionization ratio reduces the spatial shift between the beam and the waveguide, insuring an efficient coupling between the two regardless of the beam intensity. While this is indeed the case for the experimental results presented in this paper, it would be an oversimplification to generalize this conclusion. Indeed, the theoretical model suggests that the beam could be temporarily defocused before being focused (see [37], Fig. $6(\mathrm{a}))$. It is therefore clear that, in order to know the final state of the beam at the output of the crystal, one must take into account the temporal dynamic of waveguide inscription and the way it affects beam propagation. Thus, a working model must be time resolved and must include beam propagation.

Going back to the results presented here, it is clear that the ionization ratio is responsible for the presence or absence of a focusing/defocusing transition.

Note that in all previous experiments $[30,36]$ the temperature and doping where in the same range as in our experiment. The control of the ionization ratio via a background illumination is a most practical method to control the self focusing process and the presence of an intensity dependent sign reversal: unlike doping, it can be easily tuned, and is much faster than temperature tuning.

diameter is measured from a Gaussian fit, this means that the actual value of $I_{\text {inv }}$ could be considered more like a range of intensities in which the transition focusing-defocusing takes place.

${ }^{13} \mathrm{As}$ a side note, we would like to point out that the index contrast between the guide and the anti-guide is responsible for the higher than expected index variation previously measured [33]. 


\section{Conclusions}

In this paper the PR self focusing process in InP:Fe is investigated. We have systematically characterized the evolution of self focusing ratio and beam bending as a function of beam intensity, temperature, and beam illumination for two different iron dopings and showed how self focusing can be tuned by using these parameters. We have found out that the ionization ratio of the crystal is responsible for the existence or absence of a focusing/defocusing transition, thus explaining previously contradicting reports of different behaviors of the said transition $[30,36]$. Also, our results indicate that the resonance intensity is different from the inversion intensity, as predicted in [38]. The simulations obtained from a new theoretical model [37] have been compared against experimental results presented here and found to be in qualitative agreement. The results presented in this paper clarify the mechanisms responsible for PR self focusing in semiconductors in general and InP:Fe in particular, which is essential for any application involving the use of one or more self focused beam in such materials. These self-focusing and de-focusing measurements can thus be used as a basis for further spatial soliton propagation characterization.

\section{Acknowledgments}

The authors wish to thank Nicole Wolffer for her insightful suggestions, Region Lorraine for the financial support and InPact Society for the InP:Fe crystals.

\section{References}

[1] M. Segev, B. Crosignani, and A. Yariv. Spatial solitons in photorefractive media. Phys. Rev. Lett., 68(7):923, 1992.

[2] B. Crosignani, M. Segev, D. Engin, P. Di Porto, A. Yariv, and G. Salamo. Self-trapping of optical beams in photorefractive media. J. Opt. Soc. Am. $B, 10(3): 443,031993$.

[3] G. C. Duree, J. L. Shultz, G. J. Salamo, M. Segev, A. Yariv, B. Crosignani, P. Di Porto, and E. J. Sharp. Observation of self-trapping of an opical beam due to the photorefractive effect. Phys. Rev. Lett., 71(4):533, 061993.

[4] M. D. Iturbe-Castillo, P. A. Marquez-Aguilar, J. J. Sanchez-Mandragon, S. Stepanov, and V. Vysloukh. Spatial solitons in photorefractive $\mathrm{Bi}_{12} \mathrm{TiO}_{20}$ with drift mechanism of nonlinearity. Appl. Phys. Lett., 64(4):408, 011994.

[5] K. Kos, H. Meng, G. Salamo, M. Shih, M. Segev, and G. C. Valley. Onedimensional steady state photorefractive screening soliton. Phys. Rev. B, 53(5):4330, 051996. 
[6] M. Shih, M. Segev, G. C. Vally, G. Salamo, B. Crosignani, and P. DiPorto. Observation of two dimensional steady-state photorefractive screening solitons. Elec. Lett., 31:826-827, 1995.

[7] J. A. Andrade-Lucio, M. D. Iturbe-Castillo, P. A. Marquez-Aguilar, and R. Ramos-Garcia. Self-focusing in photorefractive $\mathrm{BaTi0}_{3}$. Optical and Quantum Electronics, 30:829-834, 1998.

[8] C. Anastassiou, M. F. Shih, M. Mitchell, Z. Chen, and M. Segev. Optically induced photovoltaic sel-focusing-to-self-defocusing transition. Opt. Lett., 23(12):924, 061998.

[9] S.R. Singh and D.N. Christodoulides. Evolution of spatial optical solitons in biased photorefractive media under steady state conditions. Opt. Comm., 118:569, 081995.

[10] A. A. Zozulya, D. Z. Anderson, A. V. Mamaev, and M. Saffman. Selffocusing and soliton formation in media with anisotropic nonlocal material response. Europhys. Lett., 36:419, 1996.

[11] A.A. Zozulya, D.Z. Anderson, A.V. Mamaev, and M.Saffman. Solitary attractors and low-order filamentation in anisotropic self-focusing media. Physical Review A, 57:522, 1998.

[12] M. Segev, M. Shih, and G. C. Valley. Photorefractive screening solitons of high and low intensity. Journ. Opt. Soc. Am. B, 13:706, 1996.

[13] A. A. Zozulya and D. Z. Anderson. Nonstationary self-focusing in photorefractive media. Opt. Lett., 20(8):837, 041995.

[14] N. Fressengeas, J. Maufoy, and G. Kugel. Temporal behavior of bidimensional photorefractive bright spatial solitons. Phys. Rev. E., 54:6866-6875, 1996.

[15] E. Fazio, F. Renzi, R. Rinaldi, M. Bertolotti, M. Chauvet, W. Ramadan, A. Petris, and V. I. Vlad. Screening-photovoltaic bright solitons in lithium niobate and associated single-mode waveguides. Applied Physics Letters, 85(12):2193-2195, 2004.

[16] M. F. Shih and M. Segev. Incoherent collisions between two-dimensional bright steady-state photorefractive screening solitons. Opt. Lett., 21(19):1538, 101996.

[17] H. Meng, G. Salamo, F. M. Shih, and M. Segev. Coherent collisions of photorefractive solitons. Opt. Lett., 22(7):448, 1997.

[18] W. Krolikowski, C. Denz, A. Stepken, M. Saffiman, and B. Luther-Davies. Interaction of spatial photorefractive solitons. Qun. Sem., 10:823, 1998. 
[19] C. Denz, W. Krolikowski, J. Petter, C. Weilnau, T. Tschudi, M. R. Belic, F. Kaiser, and A. Stepken. Dynamics of formation and interaction of photorefractive screening solitons. Phys. Rev. E, 60(5):6222, 1999.

[20] A. Stepken, M. R. Belic, F. Kaiser, W. Krolikowski, and B. Luther-Davies. Three dimensional trajectories of interacting incoherent photorefractive solitons. Phys. Rev. Lett., 82(3):540, 1999.

[21] M. Klotz, H. Meng, G. J. Salamo, M. Segev, and S. R. Montgomery. Fixing the photorefractive soliton. Opt. Lett., 24(2):77, 011999.

[22] Aqiang Guo, Michael Henry, Gregory J. Salamo, Mordechai Segev, and Gary L. Wood. Fixing multiple waveguides induced by photorefractive solitons: directional couplers and beam splitters. Optics Letters, 26(16), 2001.

[23] Jurgen Petter, Jochen Schroder, Denis Trager, and Cornelia Denz. Optical control of arrays of photorefractive screening solitons. Optics Letters, 28(6), 2003.

[24] M. T. Taya, M. C. Bashaw, M. Segev, and G. C. Valley. Observation of dark photovoltaic spatial solitons. Phys. Rev. A, 52(4):3095, 101995.

[25] K. Kos, G. Salamo, and M. Segev. High intensity nanosecond photorefractive spatial solitons. Opt. Lett., 23:1001, 1998.

[26] D. Wolfersberger, F. Lhommé, N. Fressengeas, and G. Kugel. Simulation of the temporal behavior of one single laser pulse in a photorefractive medium. Opt. Commun., 222:383, 2003.

[27] C. Dan, D. Wolfersberger, N. Fressengeas, G. Montemezzani, and A. A. Grabar. Near infrared photorefractive self focusing in $\mathrm{Sn}_{2} \mathrm{P}_{2} \mathrm{~S}_{6}$ crystals. Optics Express, 15(20):12777, 2007.

[28] D. Wolfersberger, N. Khelfaoui, C. Dan, N. Fressengeas, and H. Leblond. Fast photorefractive self-focusing in InP:Fe semiconductor at infrared wavelengths. Apl. Phys. Lett., 92(2):21106-1-3, 2008.

[29] M. Alonzo, C. Dan, D. Wolfersberger, and E. Fazio. Coherent collisions of infrared self-trapped beams in photorefractive InP:Fe. Applied Physics Letters, 96(12):121111, March 2010.

[30] M. Chauvet, S.A. Hawkins, G.J. Salamo, M. Segev, D.F. Bliss, and G. Bryant. Self-trapping of planar optical beams by use of the photorefractive effect in InP:Fe. Opt. Lett., 21(17):1333, 091996.

[31] G. Picoli, P. Gravey, C. Ozkul, and V. Vieux. Theory of two-wave mixing gain enhancement in photorefractive InP:Fe : A new mecanism of resonance. App. Phys., 66:3798, 1989. 
[32] G. Picoli, P. Gravey, and C. Ozkul. Model for resonant intensity dependence of photorefractive two-wave mixing in InP:Fe. Opt. Lett., 14(24):1362, 12 1989.

[33] M. Chauvet, S. A. Hawkins, G. J. Salamo, M. Segev, D. F. Bliss, and G. Bryant. Self trapping of two dimensional optical beams and light induced waveguiding in photorefractive InP at telecomunication wavelengths. App. Phys. Lett., 70:2499-2501, 1997.

[34] N. Wolffer, P. Gravey, and R. Coquillé. Numerical analysis of photorefractive InP:Fe at large fringe contrast. J. Appl. Phys., 78(11):6375, 1995.

[35] Tal Schwartz, Yaniv Ganor, Tal Carmon, Raam Uzdin, Sharon Shwartz, Mordechai Segev, and Uri El-Hanany. Photorefractive solitons and light-induced resonance control in semiconductor CdZnTe. Opt. Lett., 27(14):1229-1231, 2002.

[36] N. Fressengeas, N. Khelfaoui, C. Dan, D. Wolfersberger, G. Montemezzani, H. Leblond, and M. Chauvet. Roles of resonance and dark irradiance for infrared photorefractive self-focusing and solitons in bi-polar InP:Fe. Phys. Rev. A, 75, 2007.

[37] F. Devaux and M. Chauvet. Three-dimensional numerical model of the dynamics of photorefractive beam self-focusing in InP:Fe. Phys. Rev. A, 79:033823, 2009.

[38] H. Leblond and N. Fressengeas. The theory of photorefractive resonance for localized beams in two-carrier photorefractive systems. Physical Review A, 80(3):033837, 2009.

[39] R. Fornari and J. Kumar. Infrared absorption spectra in bulk fe-doped InP. Applied Physics Letters, 56(7):638-640, 1990.

[40] S. Shwartz, M. Segev, and U. El-Hanany. Self-deflection and all-optical beam steering in CdZnTe. Optics Letters, 29:760, 2004.

[41] G. C. Valley, S. W. McCahon, and M. B. Klein. Photorefractive measurement of photoionisation and recombination cross sections in InP:Fe. $J$. Appl. Phys., 64(12):6684-6689, 1988.

[42] B. Mainguet, F. Le Guiner, and G. Picoli. Moving grating and intrinsic electron-hole resonance in two-wave mixing in photorefractive InP:Fe. Opt. Lett, 15(17):938, 091990.

[43] C. Ozkul, G. Piccoli, P. Gravey, and N. Wolffer. High gain coherent amplification in thermally stabilized InP:Fe crystals under dc fields. Appl. Optics, 29(18):2711, 1990. 